\title{
Thermophoresis of an Aerosol Particle in a Microtube
}

\author{
Huan J. Keh, Cheng Y. Li \\ Department of Chemical Engineering, National Taiwan University \\ Taipei 10617, Taiwan \\ huan@ntu.edu.tw
}

\section{Extended Abstract}

When a solid surface in contact with a rarefied gas possesses a tangential temperature gradient, a thin Knudsen layer of the fluid adjacent to the surface will flow along with the gradient. This phenomenon is well known as thermal creep, which provides a mechanism for the thermophoresis of an aerosol particle in the slip-flow regime. Thermophoresis refers to the particle motion in response to a temperature gradient in the bulk gas against its direction. Being a mechanism for the capture of aerosol particles on cool surfaces, thermophoresis plays an important role in many practical applications such as aerosol sampling, air cleaning, microelectronic manufacturing, scale formation on heat exchanger surfaces, removal of soot particles for combustion exhaust gas systems, modified chemical vapour deposition, and nuclear reactor safety [1,2].

In most real applications of thermophoresis in microfluidic devices, the dimensions of the aerosol particles and confining microchannels are comparable, and it is needed to ascertain if the proximity of the channel wall significantly influences the particle velocity. The thermophoretic motions of a spherical particle parallel and perpendicular to a single plane wall or a pair of plane walls were examined by using the methods of boundary collocation and successive reflections $[3,4]$. Beyond that, the thermophoresis of a spherical particle in a spherical cavity was also analytically investigated [5].

In this paper, a theoretical study is presented for the axially symmetric thermophoresis of a spherical particle in a microtube filled with a gaseous medium. The uniformly applied temperature gradient is tangential to the tube wall, which is either prescribed with the linear temperature distribution or well insulated. The Knudsen number is small and the fluid motion is characterized by a continuum flow with temperature jump, thermal creep, and frictional slip at the solid surfaces. The general solution to the thermal and aerodynamic governing equations is presented in both spherical and cylindrical coordinates, and the boundary conditions at the particle surface are enforced by a collocation technique. The collocation solutions for the particle's thermophoretic velocity, which are in good agreement with the asymptotic formula resulting from the method of reflections, are obtained for different particle, tube wall, and fluid characteristics. A tube wall prescribed with the far-field temperature distribution and an insulated tube wall influence the thermophoresis of the particle differently. The mobility of a particle confined by a tube wall without thermal creep is a decreasing function of the particleto-tube radius ratio. When the thermal creep coefficients of the particle and of the tube wall are comparable, the thermoosmotic fluid flow caused by the wall strongly dominates the particle movement and can simply reverse its direction. In general, the influence of the confining tube on thermophoresis is significant.

The thermophoretic velocity of an aerosol sphere parallel to two plane walls was determined previously for different values of the relevant parameters [3]. For the cases of the insulated walls at small particle conductivity and of the walls with the prescribed temperature distribution at large particle conductivity, the velocity of the particle first decreases but then increases with an increase in the particle-to-tube radius ratio. As the particle-wall separation distance turns small, the particle mobility can even be greater than that in an unbounded fluid (by as much as $22 \%$ for a typical case). This distinction in boundary effects on thermophoresis between tube and slit channels is pronounced, indicating that the effects of hydrodynamic retardation (in comparison with that of thermal interactions) are much stronger in a tube than in a slit. The overall boundary effects on thermophoresis are generally more substantial in a tube than in a slit.

\section{References}

[1] Y. C. Chang and H. J. Keh, "Effects of thermal stress slip on thermophoresis and photophoresis," J. Aerosol Sci., vol. 50, pp. 1-10, 2012. 
[2] Y.-T. Wu, B. Yang, and Y.-P. Zhao, "Thermophoresis of aerosol particles in near-critical vapor: An inverse size effect," Appl. Phys. Lett., vol. 106, 251605, 2015.

[3] H. J. Keh and P. Y. Chen, "Thermophoresis of an aerosol sphere parallel to one or two plane walls," AIChE J., vol. 49, pp. 2283-2299, 2003.

[4] H. J. Keh and Y. C. Chang, "Thermophoresis of an aerosol sphere perpendicular to two plane walls," AIChE J., vol. 52, pp. 1690-1704, 2006.

[5] H. J. Keh and J. H. Chang, "Boundary effects on the creeping-flow and thermophoretic motions of an aerosol particle in a spherical cavity," Chem. Eng. Sci., vol. 53, pp. 2365-2377, 1998. 